\title{
Pathology of the cardiomyopathies: geographical aspects
}

\author{
M. S. R. Hutt
}

THERE are two reasons why a geographical pathologist should be involved in a discussion on cardiomyopathies. Firstly, in these days of rapid and easy transport from one part of the world to another, a clinician or pathologist working in this country may have to see patients who have spent all their lives in other parts of the world where the patterns of disease, including cardiomyopathies, are very different. Secondly, by comparing these differences in the incidence or prevalence of a disease, one may pick up clues to its aetiology.

The pattern of cardiovascular disease in middle Africa, for example, is very different from that seen in North America and Europe, and it is important to emphasize that medicine in the tropics is not solely concerned with infectious and parasitic diseases. In a series of 2500 necropsies carried out in Kampala, Uganda, there were 268 cases who had evidence of organic heart disease. The commonest type was hypertensive cardiovascular disease, though not all cases included under this heading were in cardiac failure. A considerable proportion of these hypertensives were secondary to chronic glomerulonephritis which itself occurs more frequently in Uganda and is probably related both to $P$. malariae infections and to $\beta$-haemolytic streptococci. Rheumatic heart disease is the second commonest form of organic heart disease and acute rheumatic carditis without gross valvular lesions is commonly seen; this is an important differential diagnosis when dealing with young individuals with congestive cardiomyopathy (COCM).

Endomyocardial fibrosis (EMF) constitutes $10 \%$ of all organic heart disease, and congestive cardiomyopathy (COCM), which I will refer to in pathological terms as idiopathic cardiomegaly, accounts for $5 \%$ of cases. Ischaemic heart disease is very rare in Ugandan Africans and only one or two cases of myocardial infarction are seen each year.

Endomyocardial fibrosis has a defined geographical distribution, although there have been occasional cases reported outside this distribution. The disease is endemic in most of the middle part of Africa, Southern India and Ceylon and in parts of South America. Within this distribution there appear to be areas and groups of individuals with a higher frequency. Endemic endomyocardial fibrosis, sometimes called Davies' disease, has characteristic macroscopic lesions which can usually be easily distinguished from other causes of endocardial scarring or thrombosis, though a similar picture may be seen in Löffler's endocarditis. In a typical example involving the left ventricle the lesions are in the inflow tract, usually extending from the posterior cusp of the mitral valve to the apex, with an abrupt line of demarcation at the junction of the inflow and outflow tracts. Functlonally this often produces mitral incompetence. In right ventricular EMF the whole of the apex of the right ventricle is obliterated and the conus is dilated. Involvement of the chordae of the right ventricle gives rise to tricuspid incompetence. Pathologically the disease is frequently bilateral though the clinical effects are usually dominantly on one side.

The typical heart of idiopathic cardiomegaly (COCM) macroscopically is large and heavy, due to a combination of dilatation and hypertrophy. In assessing obscure cases of cardiac disease it is important that one should measure ventricular cavity size, as in the early stages there may be dilatation with little hypertrophy and then the weight may be only slightly increased and the wall thickness may be reduced. It should also be emphasized that the heart weight must be related to body weight and a heart weight of only $350 \mathrm{~g}$ in a small person may represent a significant increase. Inspection of the endocardial surface of these dilated hearts shows rather attenuated trabeculae, sometimes with small or large thrombi in the intrabecular spaces. A group of cases, sometimes known as Becker's cardiomyopathy, has been described from South Africa, in which thrombus formation and endocardial polyps are common features. These cases are similar in most respects to those seen elsewhere in the tropics and probably should not be regarded as a distinct group. Before making a diagnosis of idiopathic cardiomegaly in a heart showing bilateral dilatation and hypertrophy, one has to exclude hypertension (Hutt, p. 767*; Oakley, p. 777*; Goodwin, p. 780*), vascular shunts, obstructive lesions, valvular disease and ischaemic diseas. On microscopy, one can exclude conditions such as myocarditis, amyloid disease, haemochromatosis and some of the systemic conditions described by $\mathrm{Dr}$ Pomerance (p. 714*). The microscopy of idiopathic cardiomegaly (COCM) has been described by $\mathrm{Dr}$ Olsen (p. 732*). Measurement of myofibre size

* These are references to this issue 
often reveals great variation and there is anisocytosis with some fibres of normal size while others are greatly hypertrophied as judged by the width and nuclear changes. Occasionally in grossly enlarged hearts the myofibres are greatly increased in width and appear vacuolated, though this appearance is usually easily distinguished from the changes of HOCM. Such changes are probably degenerative in nature and the increase in size is not just due to hypertrophy. The amount of interstitial increase in connective tissue is variable and occasionally there are focal areas of myocytolysis and fibrosis which do not appear to be ischaemic. Some reports have drawn attention to changes in the intramural coronary vessels in idiopathic cardiomegaly. These do not appear to be constant and microvasculature injection studies in Uganda have not revealed abnormalities of either the large or small vessels.

Idiopathic cardiomegaly, as just defined, is probably a pathological dumping ground in which we neither know the aetiology nor understand the pathogenesis. Pathologists can only define the aetiology if the agent, for example a virus, can be demonstrated in the lesion. There is no evidence that this has been achieved in this condition.

The question of aetiology brings one back to epidemiology, for I think that it is from epidemiological evidence that we may get some new clues as to the aetiology. It is difficult to get reliable and comparable figures from different parts of the world, partly because reliable necropsy studies are not available everywhere. What evidence there is suggests that idiopathic cardiomegaly is more commonly seen clinically and at necropsy in many tropical countries, in particular the West Indies and a large part of sub-Saharan Africa. The higher incidence in clinical reports from these areas, as opposed to necropsy surveys, may be due to over-diagnosis by the clinician and underdiagnosis by the pathologist, though in the tropics other selective factors play a role. Furthermore, within a clinical group of congestive cardiomyopathies there will be some who who turn out to have specific changes such as a myocarditis.

My experience since returning to the U.K. from Uganda suggests that idiopathic cardiomegaly may be a commoner problem than is generally realized, not only because of the large immigrant population, but also in the indigenous population. Both clinical and pathological diagnosis are complicated here by the high prevalence of ischaemic heart disease. The finding of severe coronary artery disease in a patient with gross cardiomegaly may not be cause and effect but the co-existence of two conditions.

I would conclude by suggesting that idiopathic cardiomegaly is as interesting as HOCM, that it is perhaps more common in this country than most people suggest, and that the epidemiological approach may be the one that will give us clues as to the aetiology.

\section{Bibliography}

Brockington, I.F. \& Edington, G.M. (1972) Adult heart disease in Western Nigeria. American Heart Journal, 83, 27.

DAVIES, J.N.P. (1961) The heart of Africa. Laboratory Investigations, 10, 205.

HUTT, M.S.R. (1970) Pathology of African cardiomyopathies. Pathologia et microbiologia, 35, 37.

SHAPER, A.G. (Ed.) (1968) Introduction to the Cardiomyopathies. S. Karger, Basel, Switzerland.

Miall, W.E. \& Bras, G. (1972) Heart disease in the tropics. British Medical Bulletin, 28, 79.

World Health ORganization (1965/68) Working party on cardiomyopathies. Bulletin of the World Health Organization, 33, 257, 38, 979. 